\title{
HOW TO DISTINGUISH PERFECT QUASI-CRYSTALS FROM TWINS AND OTHER STRUCTURES USING DIFFRACTION EXPERIMENTS?
}

\author{
J. WOLNY \\ Faculty of Physics and Nuclear Techniques, Academy of Mining and Metallurgy \\ Al. Mickiewicza 30, 30-059 Kraków, Poland
}

\begin{abstract}
Performing diffraction experiments for various lengths of coherent scattering and using the scaling of peak intensities on a number of atoms one can experimentally distinguish quasi-crystals from the other structures (e.g. twins or random). For perfect quasi-crystals peak intensities scale as $N^{2}$, for other structures this scaling depends on concentration of atoms, behaving critical for Penrose concentration.
\end{abstract}

PACS numbers: 61.10.-i

In this paper we compare different types of two-dimensional structures (Fig. 1) which give tenfold symmetry of calculated diffraction patterns $[1,2]$. We have studied several different structures which can be obtained by tiling the plane without defects using only two types of Robinson triangles [1-3]. These structures can generally be divided into four types: Penrose-like structures, twins, random structures, and precipitated structures. For decoration of triangles two types of atoms were used: primary (large) ones in the corners of the triangles and secondary (small) ones in positions inside the big triangles (one per triangle) [4]. The structure factor was calculated for values of atomic form factors as equal to 1 for large atoms and 0 for small atoms.

In order to obtain a Penrose-like tiling, one has to obey specific rules when decorating the plane (the so-called matching rules [5]). These rules are rather easy to obey when using the inflation method to generate a structure. In this method, the Robinson triangles can be subdivided in the following way [1, 2]: each large triangle gives two large triangles and a small one and each small triangle gives one large and one small triangle. After subdivision, the resulting triangles are similar to the Robinson triangles, but they are $\tau \cong 1.618$ times smaller. After subdividing all the triangles the whole system is inflated $\tau$ times to recover the original size of the building elements. The procedure can be applied infinitely many times allowing a non-periodic covering of the whole plane with only two types of triangles. Perfect quasi-crystals, called Penrose-like structures, have well-defined 


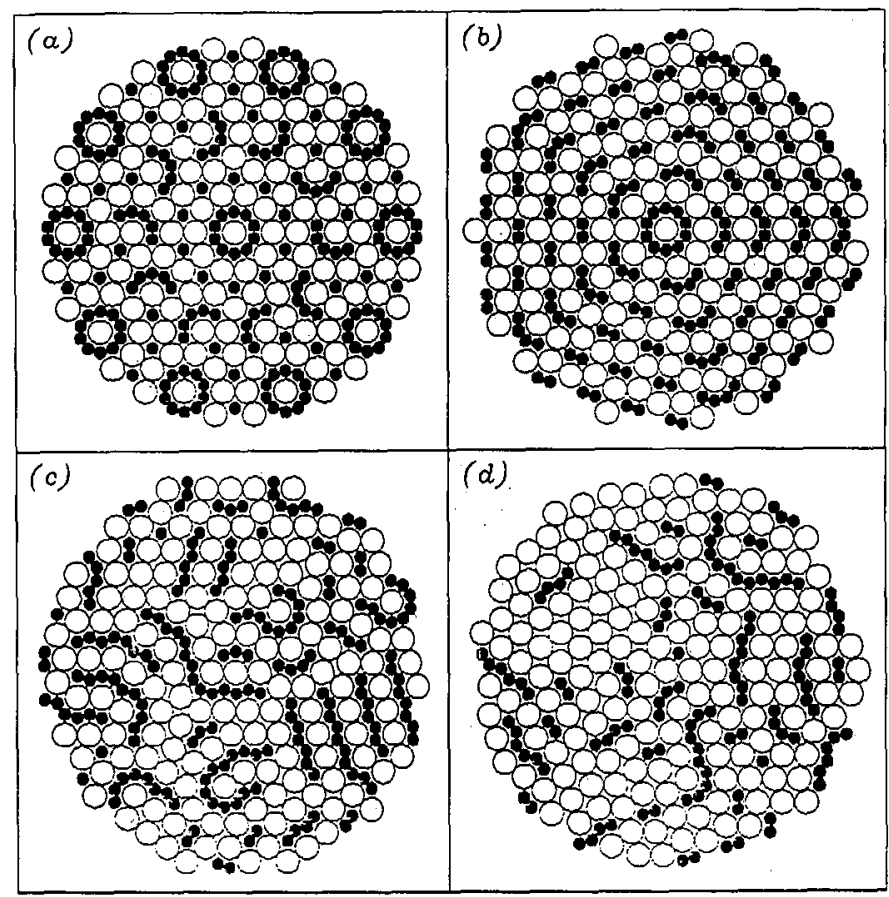

Fig. 1. Various types of structures obtained by decoration of Robinson triangles with two types of atoms: (a) Penrose-like structure; (b) a twin structure $(c=0.5)$; (c) a random structure $(c \cong 0.37)$; (d) a precipitated structure $(c \cong 0.77)$.

concentration of atoms (the critical concentration of small Robinson triangles is equal to $c_{\mathrm{p}}=1 / \tau^{2}$ ) which is constant for a chosen decoration of unit blocks (triangles) used for building of the structure.

Random and precipitated structures were generated using a computer program simulating growth of a crystal seed by random attachment of Robinson triangles under the strict condition of tiling the plane without defects [1, 2]. Random structure shown in Fig. 1c has the concentration of small Robinson triangles equal to 0.37 , the concentration which is very close to the critical one $\left(c_{\mathrm{p}} \cong 0.3812\right)$. The concentration for the precipitated structures from Fig. 1d is about 0.8 and it is much higher than the critical one. This leads to the precipitations of crystalline structures built up only with small Robinson triangles [1, 2]. Twins for different concentration of small Robinson triangles were also analyzed.

Diffraction patterns for the different structures look very similar (Fig. 2). The diffraction pattern for Penrose-like tiling contains sharp, single peaks, the intensities of which are proportional to $N^{2}$ as in classical crystallography. In the case of the twin structure, groups of peaks are observed [1] instead of single peaks. However, with increasing value of the scattering vector $k$ the peaks in each group overlap, giving a sharp intensity maximum at the Penrose position. For the random 


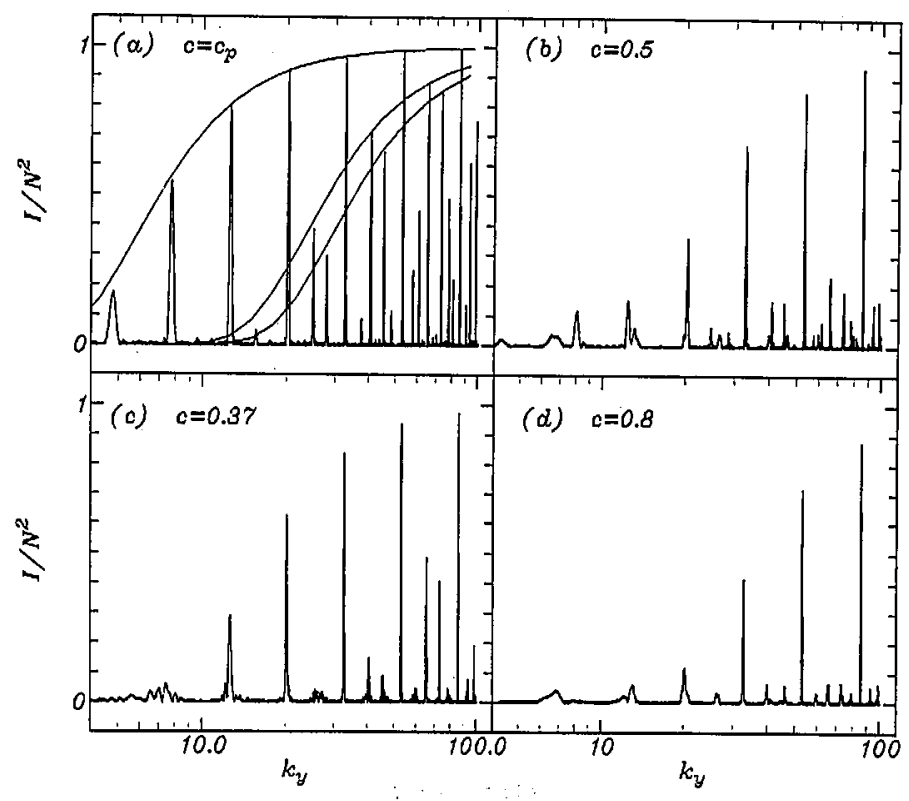

Fig. 2. Normalized diffraction patterns in the five directions equivalent to $k_{y}$ for corresponding structures shown in Fig. 1. The structure factor was calculated for values of atomic form factors as equal to 1 for large atoms and 0 for small atoms.

structure there are always peaks at the Penrose positions, but their shapes are rather complex.

As it was already shown $[2,4,6,7]$, diffraction patterns of the discussed structures can be successfully analyzed in five dimensions. In this case the intensities of peaks calculated at Penrose positions are well-approximated by the Debye-Waller factor calculated for phason-space (perp-space) fluctuations. These fluctuations are constant for Penrose-like tilings and linear in number of atoms for other structures. The slope coefficient of this relation depends linearly on deviation from Penrose concentration, where it exhibits a singular point.

For structures with concentrations different than the critical one the normalized peak intensities at Penrose-like positions are

$$
I / N^{2}=\exp (-A \Delta c N)
$$

where $I$ is the peak intensity, $\Delta c$ is the deviation of the concentration from the critical one and $N$ is the number of atoms. For a given series of peaks $[1,2]$ variable $A$ is proportional to square of the perp-space scattering vector or to one over the square of real space scattering vector. The relation (1) means that the peak intensity decreases exponentially with increasing number of atoms (Fig. 3b). However, for a given number of atoms peak intensities increase with increasing values of scattering vectors given by

$$
k_{n}=k_{0} \tau^{n}
$$




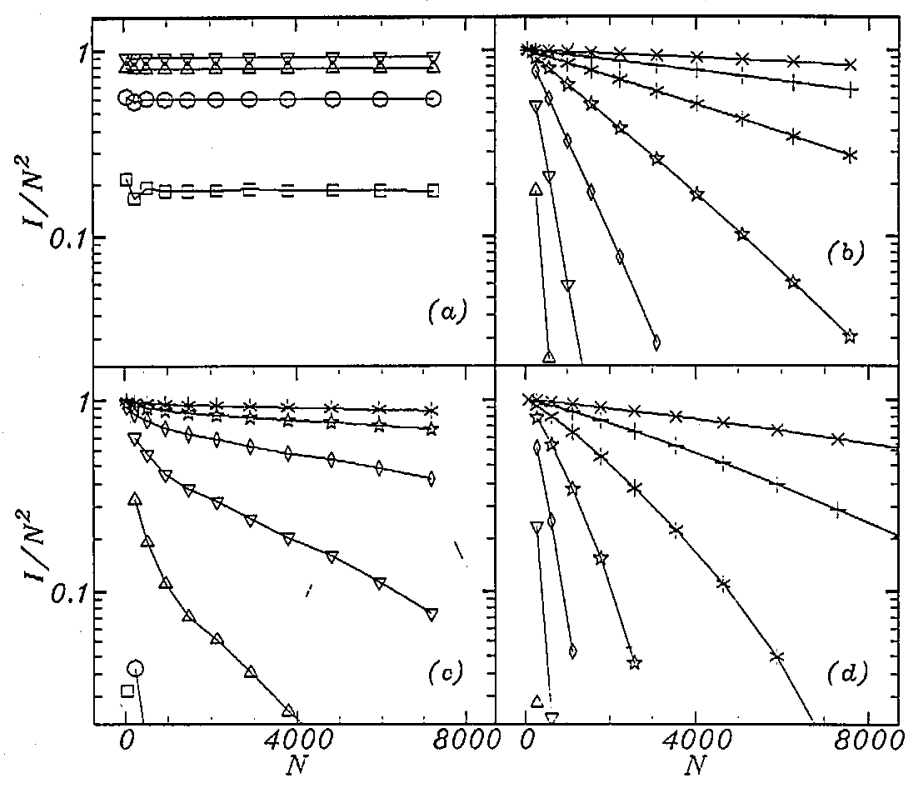

Fig. 3. Peak intensities versus number of atoms for corresponding structures shown in Fig. 1. Calculations were done for series of peaks described by Eq. (2) with peak numbers $n$ indicated as follows: $\square n=1 ; \circ n=2 ; \Delta n=3 ; \nabla n=4 ; \diamond n=5 ; \star n=6$; $* n=7 ;+n=8 ; \times n=9$.

or

$$
k_{n}^{\text {perp }}=k_{0}(-\tau)^{-n}
$$

where $n$ is an integer and values of $k_{0}$ for the first three series of peaks are constant and equal to about: $2.9545,5.9091$, and 6.6065, respectively. Peak intensities increase with $n$ approaching 1 for higher values of scattering vectors.

Random Penrose-like tilings are obtained for random arrangement of unit blocks at the critical concentration of atoms [1,2]. For this concentration the slope coefficient of the linear relation of mean square fluctuations versus number of atoms vanishes and the logarithmic term begins to dominate, changing the scaling of the peak intensities to

$$
I / N^{2}=N^{-\eta / 2} \text {, }
$$

where $\eta$ is a quadratic function of one over scattering vector given by (2). However, the above equation is not valid for three-dimensional random quasi-crystals at critical concentration [8].

The scaling of peak intensities at Penrose positions given by Eq.(2) distinguishes perfect quasi-crystals from all other structures. For Penrose-like structures peak intensities scale as $N^{2}$, for other two-dimensional structures this scaling is given by Eq. (1) or (3). This property of the diffraction pattern can be used for experimental verification of the structure. To do that, one has to measure diffraction patterns of a given structure for different numbers $N$ of coherently scattering 
atoms; their numbers depend on the length of coherent scattering in diffraction experiments. If the relative peak intensities are constant for all such diffraction patterns, the structure belongs to perfect quasi-crystals (or crystals). If not, the defects or twins are present in the structure. Comparing the diffraction patterns to the similar ones obtained for perfect crystals one can find the scaling of peak intensities versus $N$, which allows to estimate the divergency of concentration from the critical one.

Changing the length of coherent scattering is the most serious problem from the experimental point of view. When synchrotron radiation is used one can measure the diffraction patterns for different volumes of irradiated sample or different wavelengths. The absorption coefficient strongly depends on the wavelength of the X-ray (especially near the absorption edge of the scattering elements), therefore the length of coherent scattering can be changed using different wavelengths. Performing such experiment one can finally obtain the scaling of peak intensities versus number of atoms. This information is a crucial one for distinguishing perfect quasi-crystals from all other structures. For perfect quasi-crystals the relative intensities do not depend on the number of atoms, therefore they should be constant for all the wavelengths used in the experiment.

\section{References}

[1] J. Wolny, L. Pytlik, B. Lebech, J. Phys. C, Solid State Phys. 21, 2267 (1988).

[2] J. Wolny, Zesz. Nauk. AGH, Fizyka 23 (1991).

[3] C. Godreche, H. Orland, J. Phys. Coll. 47, C3, 197 (1986).

[4] J. Wolny, L. Pytlik, J. Phys., Condens. Matter 3, 2457 (1991).

[5] A. Doroba, Postępy Fiz. 40, 3 (1989).

[6] M.V. Jarić, Phys. Rev. B 34, 4685 (1986).

[7] L.-H. Tang, M.V. Jarić, Phys. Rev. B 41, 4524 (1990).

[8] M. Widom, private communication. 